\title{
REFLECTION
}

\section{The Teamlet Model of Primary Care}

Thomas Bodenbeimer, MD

Brian Yosbio Laing, BS

Department of Family and Community Medicine, University of California, San Francisco, Calif
AC Annals Journal Club selection; see inside back cover or http://www. annfammed.org/AJC/.

Conflicts of interest: none reported

\section{CORRESPONDING AUTHOR}

Thomas Bodenheimer, MD

Department of Family and Community Medicine

University of California, San Francisco Bldg 80-83, San Francisco General Hospital 995 Potrero Ave

San Francisco, CA 94110

TBodenheimer@fcm.ucsf.edu

\begin{abstract}
The 15-minute visit does not allow the physician sufficient time to provide the variety of services expected of primary care. A teamlet (little team) model of care is proposed to extend the 15-minute physician visit. The teamlet consists of 1 clinician and 2 health coaches. A clinical encounter includes 4 parts: a previsit by the coach, a visit by the clinician together with the coach, a postvisit by the coach, and between-visit care by the coach. Medical assistants or other practice personnel would require retraining to assume the health coach role. Some organizations have instituted aspects of the teamlet model. Primary care practices interested in trying out the teamlet concept need to train 2 health coaches for each full-time equivalent clinician to ensure smooth patient flow.
\end{abstract}

Ann Fam Med 2007;5:457-461. DOI: 10.1370/afm.731.

\section{INTRODUCTION}

7 he following research findings show that the central institution of primary care-the 15-minute physician visit—can no longer accomplish what society expects:

- Fifty percent of patients leave the office visit without understanding what advice their physician gave. ${ }^{1}$

- Physicians, according to 1 study, interrupted patients' initial statement of their problem in an average of 23 seconds; in $25 \%$ of visits the patient was unable to express his/her concerns at all. ${ }^{2}$

- It takes 7.4 hours per working day to provide all recommended preventive care to a panel of 2,500 patients, plus 10.6 hours to manage all chronic conditions adequately.,

- Forty-two percent of primary care physicians report not having adequate time to spend with their patients. ${ }^{5}$

During the 15 -minute visit, primary care physicians cannot provide acute, chronic, and preventive care while building meaningful relationships with their patients and managing multiple diagnoses according to a host of evidence-based guidelines. The 15 -minute physician visit must be eliminated as the central institution of primary care. The teamlet (little team) model is offered as a replacement for the 15 -minute physician visit. This model has 2 central features: (1) the patient encounter involves 2 caregivers-a clinician (physician, nurse-practitioner, or physician's assistant) and a health coach-rather than only the clinician; and (2) the 15-minute visit is expanded to include previsit, visit, postvisit, and between-visit care.

\section{WHAT IS A TEAMLET?}

All primary care practices have a team. For a small private office, the team is the physician, medical assistant, and receptionist. For community health centers, outpatient department clinics, and multispecialty groups, the team is larger and includes a variable mix of physicians, advanced practice clinicians, registered nurses, licensed vocational and practical nurses, medical 
assistants, receptionists, health educators, pharmacists, social workers, and community health workers.

Whereas the team varies dramatically with the size and type of practice, one constant feature as the central subunit of the team in almost all primary care settings is the clinician-medical assistant dyad.

The teamlet model proposes a transformation of this universal dyad. This transformed dyad is called the teamlet because it is only a small part of the primary care team. In this model, medical assistants or other appropriate personnel are retrained in such skills as chronic disease self-management support to assume the role of a health coach. Each patient cared for by the teamlet would participate in an expanded visit with both a clinician and a health coach.

The goals of the teamlet model are fivefold: (1) to improve the patient experience and enhance patients' self-management skills by expanding the encounter to include one-on-one time with a trained health coach; (2) to improve process and outcome measures for preventive and chronic care by delegating routine processes (eg, ordering periodic cholesterol measurements or mammograms based on standing orders) to health coaches and by working more intensively with patients on their chronic disease self-management skills; (3) to enhance the work life of primary care clinicians by offloading tasks that can be completed by nonclinician staff; (4) to ensure that all practice personnel are working to their fullest potential by providing additional training, crosstraining, and mentoring so that they are able to function as health coaches; (5) to cut health care costs by reducing unnecessary hospitalization and emergency department visits through intensive management of high-risk and high-utilizing patients by using health coaches to provide frequent personal contact with these patients.

\section{THE TEAMLET MODEL}

A teamlet consists of 1 clinician and 2 health coaches. Health coaches (or whatever name a practice chooses) are retrained medical assistants, community health workers, licensed practical or vocational nurses, registered nurses, or health educators - whoever is available and willing to undertake a new job. Health coaches should have cultural and language concordance with their patients. Ideally practices or clinics create a staffing ratio of 2 health coaches for 1 clinician, because health coaches spend more time with the patient than does the clinician.

In its fullest expression, the teamlet encounter involves a previsit with the health coach, a visit including both the clinician and the health coach, a postvisit with the health coach, and between-visit care provided by the health coach. Patients with relatively simple problems do not need the full previsit, postvisit, between-visit level of care.

The components of the teamlet encounter function as follows.

\section{Previsit}

The previsit is conducted by the health coach. The goals of the previsit are to offload routine work from the clinician and to improve chronic disease and prevention process measures by having health coaches order routine studies. During the previsit the health coach works with patients to negotiate the visit agenda, elicit a basic history, check on medication use, and perform indicated tests.

\section{Huddle}

Before the previsit, the health coach and clinician huddle quickly to discuss the clinical goals for the patient encounter.

\section{Agenda Setting}

Evidence shows that physicians rarely negotiate with patients concerning the medical visit agenda even though agenda setting is likely to improve the patientcenteredness of the encounter. ${ }^{6}$ Because time is a barrier to agenda setting, transferring this activity to the previsit improves the likelihood that the patient's agenda items will be addressed. To set the visit agenda, the health coach explains the clinician's agenda items and allows patients to express fully their agenda items. Having the health coach negotiate the agenda helps to minimize the unequal power relationship between physicians and patients.

\section{Medication Reconciliation}

To save clinician time, medication reconciliation can be initiated by the health coach during the previsit and completed by the clinician during the visit. For patients on multiple medications, the coach would document which prescribed medications patients are taking, and if a patient is not taking a prescribed medication, why not. This process is intended to address outpatient medication errors, which occur frequently. ${ }^{7}$ Although evidence supporting outpatient medication reconciliation for error reduction is not yet available, this activity is coming into common use.

\section{Ordering Routine Services}

Studies have found that physicians provide only $55 \%$ of recommended care and that quality indicators improve with longer visit times. ${ }^{8-10}$ To improve process measures, during the previsit the health coach would take responsibility for ordering routine chronic disease and preventive services according to standing orders 
or protocols. The health coach would explain these services to patients and order them if a patient agrees. Blood pressures, temperatures, heart rates, finger-stick glucose levels, electrocardiograms, oxygen saturation measurements, urine dipsticks, pregnancy and sexually transmitted screening tests, vision and hearing tests, immunizations, and other indicated tasks would also be included based on standing orders and the relevance of each test to a patient's history.

\section{History Taking}

The health coach may take and record a patient's history (on paper or in the electronic medical record) using specific questionnaires prepared for each common sign or symptom (sore throat, cough, abdominal pain, back pain, headache, dizziness, etc).

\section{Visit}

Once the previsit is complete, the clinician enters the examination room with the health coach. The clinician checks the patient's history and asks additional questions to clarify and deepen the history. The coach documents (on paper or in the electronic medical record) the clinician's physical findings; fills out forms; orders laboratory tests, $\mathrm{x}$-ray studies, and referrals; sends electronic prescriptions to the pharmacy or writes prescriptions for the clinician to sign; retrieves items not in the examination room; assists with procedures, and so on. In other words, the health coach assists the clinician during the visit so that the clinician can focus on cognitive work (thinking about diagnosis and management) and on building relationships with patients. Coaches would not stay in the examination room for uncomplicated visits that do not require a postvisit or when the patient is uncomfortable having the coach in the room.

\section{Postvisit}

The purposes of the postvisit are to ensure that patients understand what took place during the visit, to engage the patient in self-management skill building, and to enhance the patient's experience with the encounter. The goal is to encourage patients to be informed and to actively manage their health conditions, as such patients tend to have better outcomes than patients who are passive recipients of care. ${ }^{11}$

\section{Soliciting Patient Concerns}

Once the clinician leaves, the postvisit with the health coach begins. The coach may begin the postvisit by asking patients whether there is anything they would like to talk about. Posing this question enhances a patient-centered approach and helps the coach and patients develop a relationship that complements the clinician-patient relationship.

\section{Closing the Loop}

Ideally, an after-visit summary would be generated to recap the advice given by the clinician during the visit - what diagnostic studies to schedule, what referrals to arrange, what medications to take, what behavior changes to work on, and when to follow-up with whom. Using the after-visit summary, the health coach can apply the technique of closing the loop by asking patients to repeat back their understanding of each item of advice given during the visit. Closing the loop, which helps the $50 \%$ of patients who do not understand the clinician's advice, ${ }^{1}$ has been found to be associated with improved outcomes in patients who have diabetes. Unfortunately, this process is seldom performed. ${ }^{12}$

\section{Goal Setting}

The health coach engages patients in collaborative goal setting, negotiating an action plan regarding diet, exercise, taking medications, or other domains of the patient's life. With the coach's assistance, patients propose realistic action plans. For example, walking to work 3 days a week or eating 1 bowl of rice per meal instead of 2. Action planning is best done by health coaches; in one study, action planning by physicians took an average of 6.9 minutes, which is prohibitively time-consuming. ${ }^{13}$ Although conclusive evidence is lacking, some studies suggest that goal setting improves health-related behaviors. ${ }^{14}$ The American Diabetes Association and American Heart Association recommend setting goals collaboratively with patients.

\section{Navigating the System}

In a final postvisit activity, the health coach makes sure that patients are able to navigate the health system to accomplish the items on the after-visit summary.

\section{Between Visits}

Strong evidence supports regular follow-up as necessary to sustain improved chronic disease outcomes ${ }^{15}$; such follow-up is the function of between-visit patient contact.

Between each visit, the health coach telephones or e-mails patients to see how they are doing, helps patients find solutions to difficulties they are having, reinforces items on the after-visit summary, and acts as a liaison between patient and clinician. The health coach keeps a between-visit log to track when each patient requires follow-up contact. An electronic medical record program could also send automatic follow-up reminders to the coach. Health coaches need a dedicated half-day per week to perform their betweenvisit responsibilities.

Patients often have difficulty reaching their clinician by telephone. In the teamlet model, patients can call 
their health coach for questions or concerns about the after-visit summary or self-management goals. For more complex questions, the health coach consults with the clinician or asks the clinician to telephone the patient.

\section{POTENTIAL CHALLENGES}

The teamlet model is not without challenges, including those associated with workflow, continuity of care, team dynamics, and training.

Maintaining a smooth workflow in the teamlet model requires a delicate balance. Clinician throughput may exceed coaches' throughput, or vice versa, depending on the complexity of a patient's needs. These logistical problems have been successfully addressed by some primary care practices that have implemented aspects of the teamlet model. ${ }^{16}$ One practice uses a ratio of 5 coaches per 2 clinicians rather than a 2 to 1 ratio. This practice also utilizes headsets and walkie-talkies to help clinicians and coaches signal each other to smooth out previsit, visit, and postvisit transitions.

Continuity of care is another challenge. It may not be possible to schedule the same coach and clinician for each patient. Practices may decide to emphasize continuity between patient and clinician, with different health coaches involved with a particular patient. Academic clinical practices with part-time faculty physicians and residents may choose to encourage continuity between the health coach and the patient. Scheduling systems need to conform to the scheduling priorities agreed upon by the practice and by the preferences of each patient.

Some physicians who have critiqued the teamlet model point out that the health coach may perform tasks that clinicians enjoy doing, such as talking with patients about behavior change. The teamlet, however, is highly flexible. If a clinician wants to talk with a patient about behavior change, the clinician is free to do so, and the health coach can be freed up for telephone calls or can begin a previsit with another patient. One purpose of the brief huddles before each patient encounter is to clarify the clinician-coach division of labor for that encounter.

The effect of a third person in the clinician-patient visit is not well known. It is possible that some patients may feel overwhelmed with 2 caregivers in the room. In at least 2 health systems, patients have appreciated the medical assistant participating in the visit. ${ }^{16}$

Medical assistants, the fastest growing sector of caregivers in the primary care workforce, ${ }^{17}$ vary in their capacity to perform the health coach function. Several primary care organizations have provided intensive training in teamlet tasks to medical assistants, generally with positive results. ${ }^{16}$
The enthusiasm and job retention of teamlet coaches can pose substantial problems. Medical assistants with certain job expectations may not wish to take on the added responsibilities, and medical assistants who have received additional training may leave to seek advanced medical training.

Teamlet-patient relationships can create tricky situations. For example, patients might share a piece of information with the coach but do not want the coach to tell the clinician. Or patients might tell the coach that they disagree with the clinician's care plan. The coach and clinician need to work out ground rules so that they do not undermine each other or break a patient's trust.

\section{TRAINING HEALTH COACHES}

It is likely that health coaches have previously been working as medical assistants, community health workers, or licensed practical or vocational nurses. In all cases, they require considerable training, which can take place during a series of lunchtime seminars or on protected half days. Training could be conducted by clinicians within a practice or by an outside master trainer experienced in this role. Equally important is mentoring, during which trainers observe and provide feedback to health coach trainees working with patients.

Training curricula for health coaches are being developed and include such topics as the difference between acute and chronic care, the importance of the caregiver-patient partnership for chronic disease, closing the loop, shared decision making, discussing behavior change action plans with patients, how to navigate the particular health system in which the teamlet model is being introduced, issues related to medication reconciliation and medication adherence, and knowledge about the specific chronic conditions that are often encountered in the practice's patient population. ${ }^{18}$ A number of primary care practices have successfully trained and mentored medical assistants in these areas. ${ }^{16}$ With time, more health professionals (clinicians, nurses, health educators, social workers, behavioral health professionals) could become master trainers who can conduct this training.

\section{FINANCIAL SUSTAINABILITY OF THE MODEL}

The teamlet model may not be financially viable under current primary care payment policy. Payment is relatively low for primary care, usually provided only for clinician services, and chiefly based on productivity (quantity of visits or relative value units). The model 
is financially sustainable only if (1) it can show payers (Medicare, Medicaid, commercial health plans) that the model can reduce total health care costs (eg, hospitalizations, emergency department visits) for high-cost patients and payers share those savings with primary care practices, or (2) the model can increase productivity under fee-for-service payment. Two organizations using a 2 to 1 ratio of medical assistants to clinicians have shown a positive business case for these additional support personnel by increasing clinician productivity. ${ }^{16}$

\section{IMPLEMENTING PARTS OF THE MODEL}

The teamlet model is a novel blueprint for addressing some of the serious problems facing primary care: inadequate visit time to provide all recommended acute, chronic, and preventive care; physician and patient dissatisfaction with the rushed atmosphere of many visits; and the inadequate quality of care provided by stressed primary care practices. A number of organizations have adopted elements of the teamlet model $_{i}$ few, if any, have developed the concept in its entirety. Implementing teamlets requires an increase in the number of medical assistants (the most likely caregivers to become health coaches) and time and expertise to train medical assistants to become coaches.

Although the teamlet model appears on the surface to be difficult to implement, practice pilot programs can be designed with 1 clinician and 2 medical assistants for 1 or 2 half-days per week. Moreover, pilot programs can begin by adding a previsit, by including the medical assistant in the visit, or by initiating a postvisit ${ }_{i}$ it is neither necessary nor desirable to launch the entire model at one time. Currently pilot projects are underway to test the feasibility of the teamlet model.

To read or post commentaries in response to this article, see it online at http://www.annfammed.org/cgi/current/full/5/5/457.

Submitted November 30, 2006; submitted revised May 12, 2007; accepted May 17, 2007.

Key words: Primary health care; delivery of health care; health care team; organizational innovation

\section{References}

1. Roter DL, Hall JA. Studies of doctor-patient interaction. Annu Rev Public Health. 1989;10:163-180.

2. Marvel MK, Epstein RM, Flowers K, Beckman HB. Soliciting the patient's agenda: have we improved? JAMA. 1999;281(3):283-287.

3. Yarnall KS, Pollak KI, Ostbye T, Krause KM, Michener JL. Primary care: is there enough time for prevention? Am J Public Health. 2003;93(4):635-641.

4. Ostbye T, Yarnall KS, Krause KM, Pollak KI, Gradison M, Michener $\mathrm{JL}$. Is there time for management of patients with chronic diseases in primary care? Ann Fam Med. 2005;3(3):209-214.

5. Center for Studying Health System Change. Physician Survey. http:// CTSonline.s-3.com/psurvey.asp.

6. Baker LH, O'Connell D, Platt FW. "What else?" Setting the agenda for the clinical interview. Ann Intern Med. 2005;143(10):766-770.

7. Institutes of Medicine. Preventing Medication Errors. Washington DC: National Academies Press; 2007.

8. McGlynn EA, Asch SM, Adams J, et al. The quality of health care delivered to adults in the United States. N Engl J Med. 2003;348(26):2635-2645.

9. Campbell SM, Hann M, Hacker J, et al. Identifying predictors of high quality care in English general practice: observational study. BMJ. 2001;323(7316):784-787.

10. Wilson A, Childs $S$. The relationship between consultation length, process and outcomes in general practice: a systematic review. $\mathrm{Br} J$ Gen Pract. 2002;52(485):1012-1020.

11. Heisler M, Bouknight RR, Hayward RA, Smith DM, Kerr EA. The relative importance of physician communication, participatory decision making, and patient understanding in diabetes self-management. J Gen Intern Med. 2002;17(4):243-252.

12. Schillinger D, Piette J, Grumbach K, et al. Closing the loop: physician communication with diabetic patients who have low health literacy. Arch Intern Med. 2003;163(1):83-90.

13. MacGregor K, Handley M, Wong S, et al. Behavior-change action plans in primary care: a feasibility study of clinicians. J Am Board Fam Med. 2006;19(3):215-223.

14. Handley M, MacGregor K, Schillinger D, Sharifi C, Wong S, Bodenheimer $\mathrm{T}$. Using action plans to help primary care patients adopt healthy behaviors: a descriptive study. J Am Board Fam Med. 2006;19(3):224-231.

15. Bodenheimer T. Helping patients improve their health-related behaviors: what system changes do we need? Dis Manag. 2005; 8(5):319-330.

16. Bodenheimer T. Building Teams in Primary Care: Lessons from 15 Case Studies. Oakland, Calif: California Healthcare Foundation; 2007. http:// www.chcf.org/documents/chronicdisease/BuildingTeamsInPrimaryCareCaseStudies.pdf

17. Tache S, Chapman S. The expanding roles and occupational characteristics of medical assistants: overview of an emerging field in allied health. J Allied Health. 2006;35(4):233-237.

18. Bodenheimer T, Davis C, Holman H. Helping patients adopt healthier behaviors. Clin Diabetes. 2007;25(2):66-70. 